\title{
22-jähriger Mann mit Verdacht auf Lungenkrebs
}

\section{2-Year-Old Man with Suspected Lung Cancer}

\section{Autoren}

Leonie Biener, Daniel Thomas, Yuri Tolkach, Dirk Skowasch

Institut

Medizinische Klinik II, Kardiologie, Pneumologie und

Angiologie, Universitätsklinikum Bonn

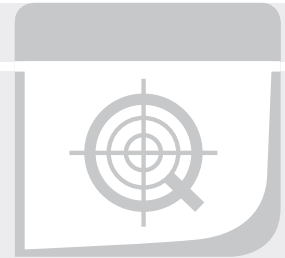

Bibliografie

DOI https://doi.org/10.1055/a-0740-0117

Dtsch Med Wochenschr 2019; 144: 13-14

(c) Georg Thieme Verlag KG, Stuttgart - New York

ISSN 0012-0472

\section{Korrespondenzadresse}

Dr. med. Leonie Biener

Universitätsklinikum Bonn

Klinik für Kardiologie, Pneumologie und Angiologie,

Sigmund-Freud-Str. 25, 53127 Bonn

Leonie.Biener@ukbonn.de
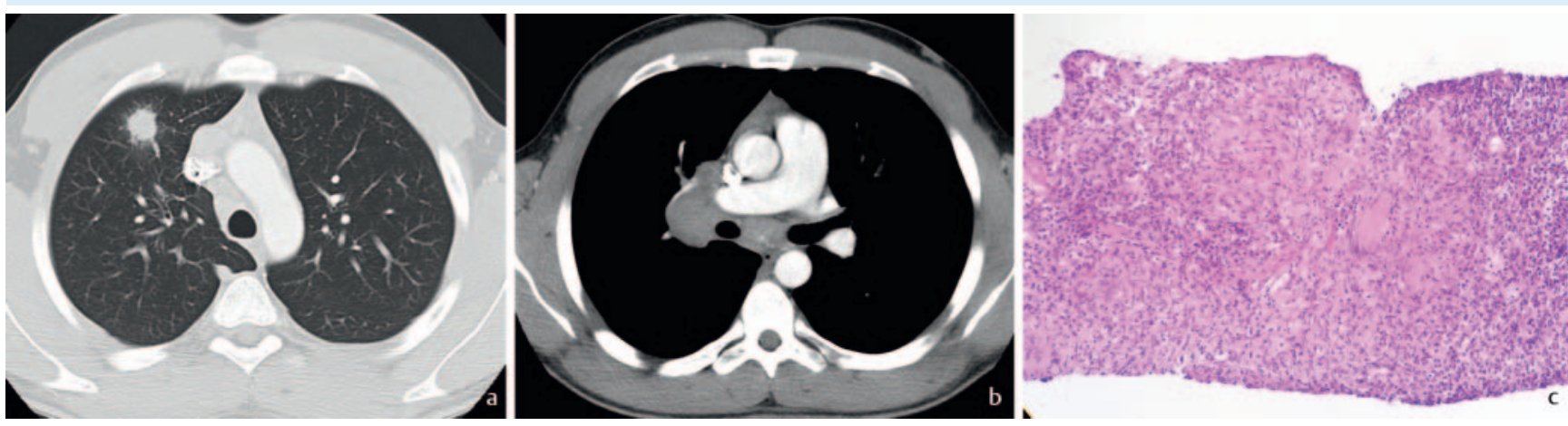

- Abb. 1 a Computertomografie des Thorax im Lungenfenster, axiale Schichtführung. b Computertomografie des Thorax im Weichteilfenster, axiale Schichtführung. c Mikroskopische Aufnahme der entnommenen Gewebeprobe.

Ein 22-jähriger Nichtraucher wurde mit dem radiologischen Verdacht auf Lungenkrebs vorgestellt. Klinisch bestehen Nachtschweiß, Gewichtsverlust ( $-4 \mathrm{~kg}$ in 2 Wochen) und abendliches Fieber. Aufgrund eines auffälligen Röntgen-Thorax-Befundes wird eine Computertomografie der Lunge veranlasst ( $\mathbf{A} \mathbf{b} \mathbf{b} \mathbf{b} \mathbf{1} \mathbf{1 a}, \mathbf{b})$, außerdem wurde eine Gewebeprobe entnommen ( $\bullet$ Abb. 1c). Die Abbildungen weisen jeweils einen pathologischen Befund auf.

\section{FRAGEN}

- Welche Befunde sind es?

- Erlauben diese Befunde eine Diagnose? Wenn ja, welche?

- Sind Differenzialdiagnosen möglich? Wenn ja, welche? 


\section{2-jähriger Mann mit Verdacht auf Lungenkrebs}

\section{2-Year-Old Man with Suspected Lung Cancer}
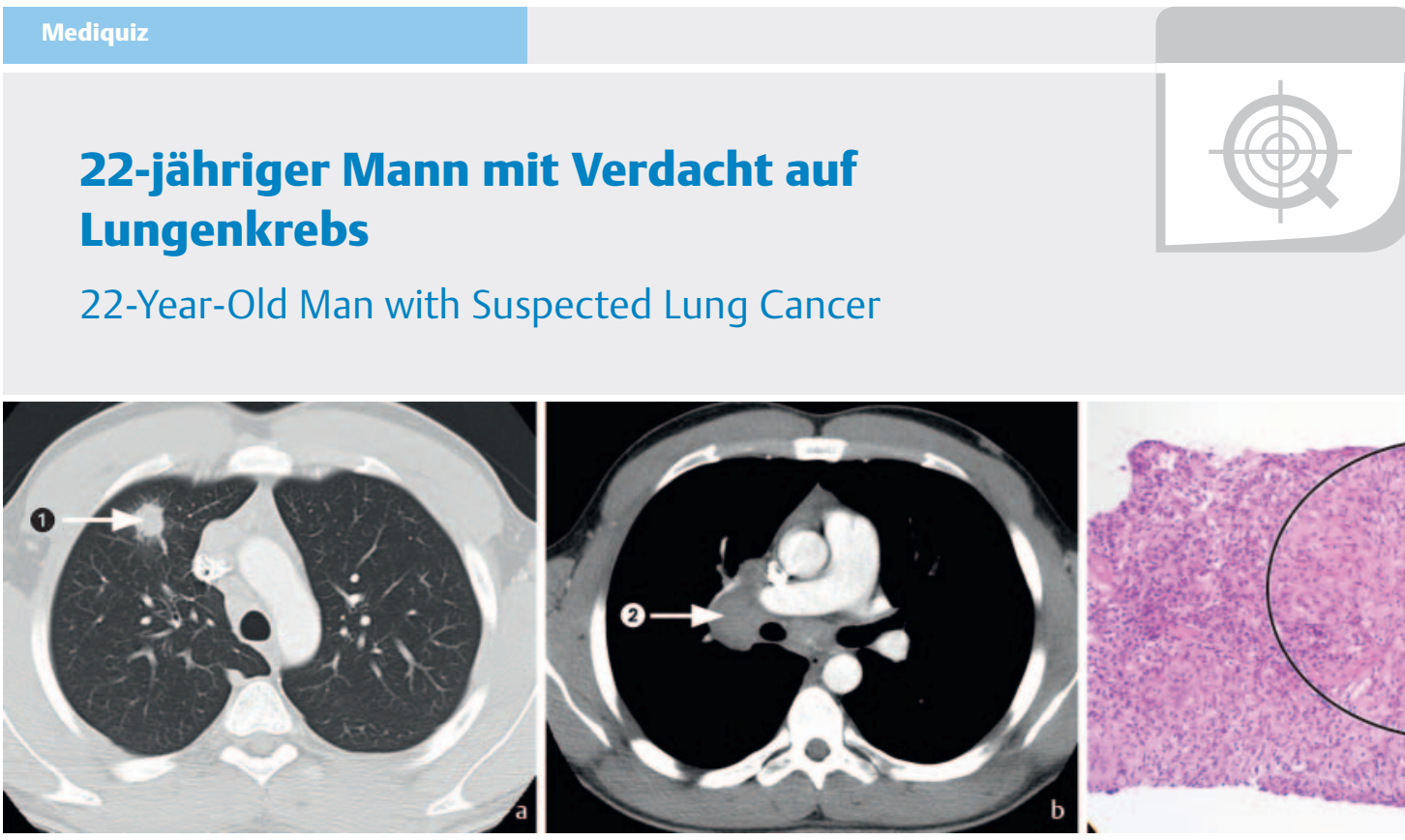

- Abb.2 a Computertomografie des Thorax im Lungenfenster, axiale Schichtführung. b Computertomografie des Thorax im Weichteilfenster, axiale Schichtführung. c Mikroskopische Aufnahme der entnommenen Gewebeprobe.

\section{Befunde}

1. Pulmonaler Rundherd im rechten Oberlappen

2. Vergrößerte rechts perihiläre und infrakarinale Lymphknoten

3. Nicht-verkäsendes Granulom

\section{Diagnose}

- Sarkoidose

\section{Differenzialdiagnose}

- Lymphom

- Tuberkulose

- Bronchialkarzinom

- Pilz-Pneumonie

\section{Erläuterung}

Die Diagnose wurde mittels einer Biopsie der Lymphknoten sowie des Lungenrundherdes gestellt. Die Sarkoidose ist eine systemische, chronisch-entzündliche Erkrankung ungeklärter Ätiologie. Die Granulome können sich in jedem Organ bilden. Wie in diesem
Fall sind häufig die Lymphknoten und die Lunge befallen; typischerweise liegt radiologisch eine bihiläre symmetrische Lymphadenopathie vor. Die Bildgebung der Sarkoidose kann, wie auch die Erkrankung selbst, sehr heterogen aussehen. Bei einseitiger Hilusvergrößerung - wie im vorliegenden Fall - ist vor allem ein Bronchialkarzinom in Erwägung zu ziehen. Daher erfolgte die Biopsie beider pathologischer Befunde. Ein Organbefall des Herzens, der Augen, der Leber, der Nieren und der Milz werden regelhaft untersucht. Eine akute Sarkoidose heilt oft selbstständig aus [1]. Liegt wie hier ein Organbefall vor, wird meist eine immunsuppressive Therapie eingeleitet [1]. Der vorgestellte Patient wurde mit einer systemischen Steroid-Therapie behandelt. Hierunter waren seine Beschwerden schnell regredient. Die Steroid-Dosis kann nach 1 - 3 Monaten bei gutem Ansprechen reduziert werden und wird je nach Therapieerfolg über etwa ein Jahr fortgeführt. Das primäre Ziel ist eine Remission der Sarkoidose, manche Patienten benötigen eine dauerhafte Therapie [1].

\section{Literatur}

[1] Pizzaro C, Skowasch D, Grohé C. Aktuelle Therapieansätze der Sarkoidose. Dtsch Med Wochenschr 2017; 142: 17-23 